\title{
Measuring oxidative burden and predicting pharmacological response in coronary artery disease patients with a novel direct activator of haem- free/oxidiseds GC
}

Citation for published version (APA):

Ahrens, I., Habersberger, J., Baumlin, N., Qian, H., Smith, B. K., Stasch, J-P., Bode, C., Schmidt, H. H. H. W., \& Peter, K. (2011). Measuring oxidative burden and predicting pharmacological response in coronary artery disease patients with a novel direct activator of haem-free/oxidiseds GC. Atherosclerosis, 218(2), 431-434. https://doi.org/10.1016/j.atherosclerosis.2011.06.042

Document status and date:

Published: 01/10/2011

DOI:

10.1016/j.atherosclerosis.2011.06.042

Document Version:

Publisher's PDF, also known as Version of record

Document license:

Taverne

Please check the document version of this publication:

- A submitted manuscript is the version of the article upon submission and before peer-review. There can be important differences between the submitted version and the official published version of record. People interested in the research are advised to contact the author for the final version of the publication, or visit the DOI to the publisher's website.

- The final author version and the galley proof are versions of the publication after peer review.

- The final published version features the final layout of the paper including the volume, issue and page numbers.

Link to publication

\footnotetext{
General rights rights.

- You may freely distribute the URL identifying the publication in the public portal. please follow below link for the End User Agreement:

www.umlib.nl/taverne-license

Take down policy

If you believe that this document breaches copyright please contact us at:

repository@maastrichtuniversity.nl

providing details and we will investigate your claim.
}

Copyright and moral rights for the publications made accessible in the public portal are retained by the authors and/or other copyright owners and it is a condition of accessing publications that users recognise and abide by the legal requirements associated with these

- Users may download and print one copy of any publication from the public portal for the purpose of private study or research.

- You may not further distribute the material or use it for any profit-making activity or commercial gain

If the publication is distributed under the terms of Article 25fa of the Dutch Copyright Act, indicated by the "Taverne" license above, 


\title{
Measuring oxidative burden and predicting pharmacological response in coronary artery disease patients with a novel direct activator of haem-free/oxidised sGC
}

\author{
Ingo Ahrens ${ }^{a, b, *}$, Jonathon Habersberger ${ }^{a, c}$, Nadège Baumlin ${ }^{a}$, Hongwei Qian ${ }^{a}$, Belinda K. Smith ${ }^{a, c}$, \\ Johannes-Peter Stasch ${ }^{\mathrm{d}}$, Christoph Bode ${ }^{\mathrm{b}}$, Harald H.H.W. Schmidt ${ }^{\mathrm{c}, \mathrm{e}}$, Karlheinz Peter ${ }^{\mathrm{a}, \mathrm{c}}$ \\ a Atherothrombosis \& Vascular Biology, Baker IDI Heart and Diabetes Institute, Melbourne, Australia \\ ${ }^{\mathrm{b}}$ Department of Cardiology and Angiology, University Hospital of Freiburg, Germany \\ c Monash University, Melbourne, Australia \\ d Bayer Schering Pharma AG, Cardiology Research, Pharma Research Centre, Wuppertal, Germany \\ e Department of Pharmacology, Maastricht University, Maastricht, The Netherlands
}

\section{A R T I C L E I N F O}

\section{Article history:}

Received 3 February 2011

Received in revised form 20 June 2011

Accepted 21 June 2011

Available online 30 June 2011

\section{Keywords:}

sGC

Platelets

Oxidative stress

Cinaciguat

BAY 58-2667

Coronary artery disease

\begin{abstract}
A B S T R A C T
Objective: The soluble guanylate cyclase (sGC) activator Cinaciguat (BAY 58-2667) represents a novel class of drugs that selectively activate oxidised sGC. The extent of oxidised sGC depends on the patient's oxidative burden. We here describe two platelet-based assays that allow determining the extent of oxidised sGC and thus provide a basis for an individualised pharmacotherapy.

Methods/Results: Platelets obtained from patients with $(n=12)$ and without $(n=12)$ coronary artery disease (CAD) were examined by flow cytometry (P-selectin expression), and Western blots (vasodilator associated phosphoprotein, VASP-phosphorylation). Results were compared to maximal oxidation of sGC achieved by the oxidising agent ODQ (1H-[1,2,4] oxadiazole[4,3-a]quinoxalin-1-one).

Treatment of platelets with Cinaciguat resulted in differential activation of oxidised sGC. Platelet P-selectin expression and VASP-phosphorylation revealed significant differences $(p=0.012, p=0.039$, respectively) between CAD and non-CAD patients.

Conclusion: We describe platelet-based assays that allow the determination of patients' oxidative status and thus allow the prediction of pharmacological response to direct sGC activators.
\end{abstract}

(c) 2011 Elsevier Ireland Ltd. All rights reserved.

\section{Introduction}

NO donors are an important component of cardiovascular pharmacotherapy in patients with atherosclerosis. However, the development of nitrate tolerance and non-selective vasorelaxation caused by nitrates is a particular limitation of chronic therapy. Recently a new class of drugs has been developed, which directly activates soluble guanylate cyclase (sGC). These drugs have the potential to overcome both limitations by directly activating SGC independently of NO and by preferentially acting on haemfree/oxidised sGC, thereby predominantly causing vasorelaxation in diseased vasculature [1]. Cinaciguat (BAY 58-2667) is the prototype of this novel class of direct haem-independent sGC activators exhibiting a strong activating effect on haem-free/oxidised sGC [1-3].

\footnotetext{
* Corresponding author at: Department of Cardiology and Angiology, University Hospital of Freiburg, Hugstetter Street 55, 79106 Freiburg, Germany. Tel.: +49 761 270 34010; fax: +49 76127070420.

E-mail address: ingo.ahrens@uniklinik-freiburg.de (I. Ahrens).
}

Haem-free/oxidised sGC typically constitutes a small percentage (approximately $2 \%$ ) of the total sGC in healthy individuals [4]. This increases in response to reactive oxygen species (ROS), which leads to oxidation of the haem iron from $\mathrm{Fe}^{2+}$ to $\mathrm{Fe}^{3+}[1]$ and also to the displacement of the haem moiety from the enzyme [4]. The oxidation process renders SGC inactive and unable to respond to its natural ligand $\mathrm{NO}$, a process that occurs in diseased atherosclerotic vessels [1]. Therefore, specifically targeting the haem-free/oxidised sGC can overcome NO resistance and has the unique potential of selectively inducing vasorelaxation in diseased vessels (Fig. 1a). Cinaciguat is currently clinically trialled for intravenous therapy of acute decompensated heart failure. Several further clinical indications such as chronic heart failure, pulmonary hypertension and coronary artery disease (CAD) are anticipated based on promising preclinical and clinical data [5,6].

Based on the pharmacological mechanism of action with a direct dependency on the availability of haem-free/oxidised sGC, a variable response in patients may be expected. We aimed to assess the potential reactivity of a patient towards a treatment with Cinaciguat and simultaneously measuring the vascular oxidative burden. For this purpose, we selected platelet sGC as an "oxidative 

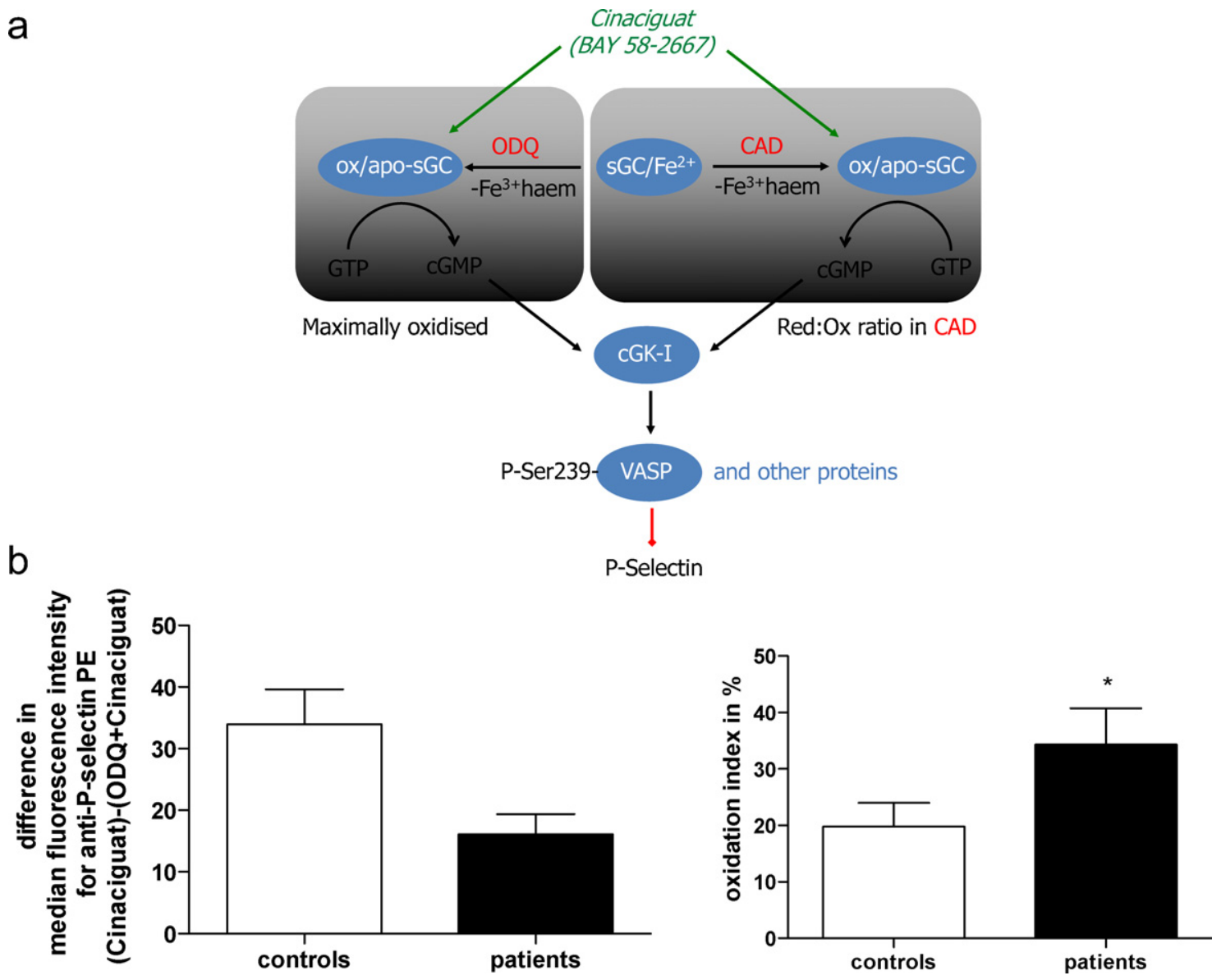

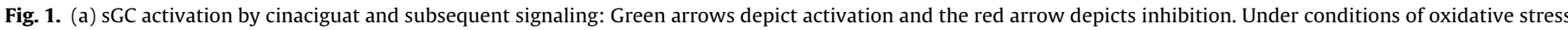

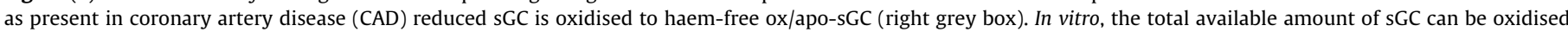

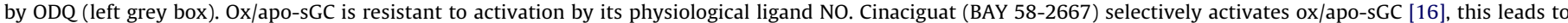

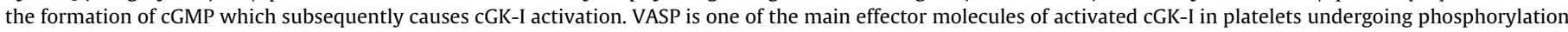

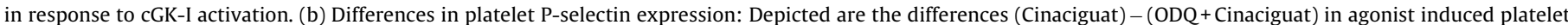

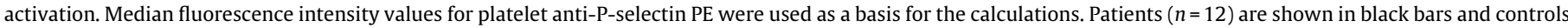

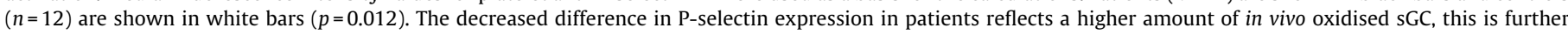

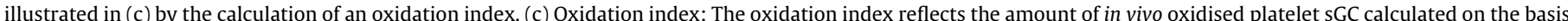

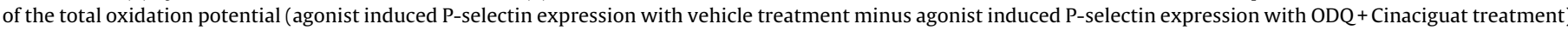

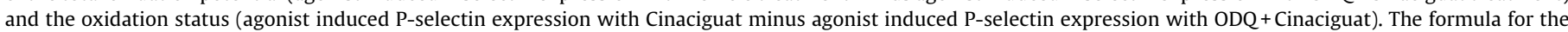
calculations was $100-$ (oxidation status/oxidation potential $) \times 100$. Patients $(n=12)$ are shown in black bars and controls $(n=12)$ are shown in white bars $(p=0.039)$.

memory" for ROS generation in the circulation. Platelets can easily be obtained from a small volume of whole blood samples and in contrast to other cells, contain only sGC and not the membrane bound particulate guanylate cyclase (pGC) [7]. Furthermore, they lack endogenous NO synthetase (NOS) [8], which minimizes the effect of sample processing on the activity of the NO receptor sGC.

Platelets were obtained from patients with CAD and control patients and incubated ex vivo with Cinaciguat to activate haemfree/oxidised sGC. Activated sGC catalyses the synthesis of the intracellular second messenger cGMP, which then activates CGMPdependent protein kinase I (cGKI). Activation of cGKI in platelets inhibits agonist-induced platelet granule secretion, calcium signaling, cytoskeletal reorganization and integrin activation [7], all crucial steps in platelet activation in vivo. Therefore, we hypothesised that agonist induced platelet activation would be inhibited in the presence of Cinaciguat, if sGC oxidation occurs as a consequence of oxidative stress in patients with CAD.

\section{Methods}

A detailed description of the methods is supplied in the online supplement.

\section{Results}

\subsection{Patient characteristics}

A total of 24 individuals admitted to undergo coronary angiography at the Heart Centre of The Alfred Hospital (Melbourne, Australia) were included in the study after the provision of written informed consent. Patients with CAD were defined by at least one vessel $>50 \%$ stenosis, controls were selected on the basis of a negative coronary angiography. The mean age was $63.7 \pm 14.2$ and $57.8 \pm 8.3$ in the patient and control groups, respectively $(p=0.24)$. The majority of patients in both groups were male $(75 \%$ of the patients and $67 \%$ of the controls). Within the patient group, the majority presented with unstable angina and a 3-vessel coronary artery disease (67\% 3 vessel disease, 25\% 2 vessel disease and 8\% 1 vessel disease).

\subsection{Flow cytometry to assess platelet reactivity}

It is known that activation of sGC reduces or inhibits platelet reactivity. Treatment of platelets with Cinaciguat resulted in markedly decreased response to ADP stimulation, which was 
stronger in patients with CAD. This was even more pronounced in the presence of ODQ $(1 \mathrm{H}-[1,2,4]$ oxadiazole[4,3-a]quinoxalin-1one), which was used to selectively oxidise sGC (data not shown). Subtracting the median fluorescence values for P-selectin expression on the platelet surface obtained in the presence of ODQ from the values obtained with Cinaciguat alone resulted in a significant difference between the control group as compared to the patient group (Fig. 1b, controls: $33.99 \pm 5.6$ vs. patients: $16.1 \pm 3.3$, $p=0.012$ ). Higher values reflect a greater amount of non-oxidised SGC that was transformed into oxidised SGC ex vivo in the presence of ODQ. Using the monoclonal antibody PAC-1, which detects GPIIb/IIIa activation, as an additional marker of platelet activation a similar trend could be seen (data not shown). Based on the data obtained with P-selectin, an oxidation index was calculated reflecting the amount of oxidised sGC in the control and patient group, respectively (Fig. $1 c$, controls: $19.76 \pm 4.2$ vs. patients: $34.29 \pm 6.5$, $p=0.039$ ). This indicates that patients with coronary artery disease have a higher oxidative burden causing increased in vivo oxidation of sGC.

\subsection{Platelet VASP phosphorylation}

Activating sGC leads to the formation of the second messenger cyclic guanosine monophosphate (cGMP). Increased intracellular cGMP then acts via cGMP-dependent protein kinases (cGK) to regulate vascular tone and blood pressure. One particular effect mediated by the cytosolic cGK, also termed cGK type I (cGKI), is the phosphorylation of VASP preferentially at serine 239 (Fig. 1a). Cinaciguat (BAY 58-2667) is a selective activator of the haemfree/oxidised sGC. Therefore, we assessed platelet VASP phosphorylation in response to Cinaciguat to determine the amount of oxidised sGC activated by Cinaciguat (Supplementary Fig. S1).

Similar to the approach used for the above described P-selectin read out, we determined the ratio of Cinaciguat induced VASP phosphorylation in the presence or absence of ODQ. We expected this ratio to be higher in patients because according to our hypothesis patients would already have more oxidised SGC and indeed we found a statistically significant difference in the SGC phosphorylation ratios (Fig. $2,0.51 \pm 0.08$ patients vs. $0.71 \pm 0.04$ controls, $p=0.039$ ).

\section{Discussion}

Using newly developed, widely accessible platelet-based assays, we can provide major advances in two directions: firstly, we show that patients with coronary artery disease have an elevated oxidative status compared to healthy controls. Secondly, we describe a novel approach that allows the identification of patients, who will benefit strongest from a highly promising new class of drugs with the potential to overcome nitrate tolerance and a mechanism of action that favors selective activation of oxidised sGC in diseased vessel areas.

Assuming that increased ROS production would be accompanied by increased generation of oxidised sGC, we tested the hypothesis that novel direct activators of haem-free/oxidised SGC such as Cinaciguat provide a unique tool to directly assess the oxidative stress status in humans. Indeed, the results of our study indicate that a portion of the platelet pool of sGC can undergo oxidative modification in vivo, which renders it inactive and resistant against its natural ligand NO. Modification of platelet sGC by superoxide has been demonstrated earlier in an in vivo system [9] and increased levels of superoxide have been found in atherosclerotic vessels in a rabbit model of atherosclerotic disease [10]. The oxidating agent (ODQ) can be used to transfer all sGC towards the oxidised form and thus provides a measure of the total amount of
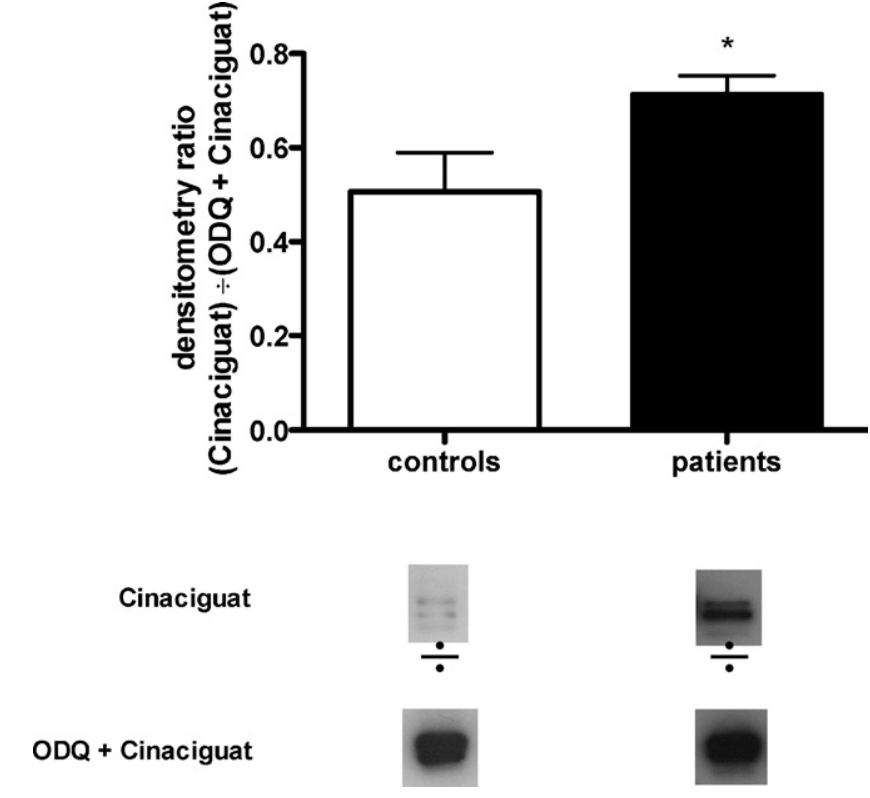

Fig. 2. Selective activation of oxidised sGC leads to phosphorylation of platelet VASP. Phosphorylation of platelet VASP was assessed by Western blot and quantified by densitometry. Phosphorylation of VASP was induced ex vivo by adding Cinaciguat (BAY 58-2667) to washed platelets in the presence or absence of ODQ. The ratio of (Cinaciguat) $-(\mathrm{ODQ}+$ Cinaciguat $)$ was used as a basis for the calculations. Depicted are patients $(n=12)$ in black bars and controls $(n=12)$ in white bars $(p=0.039)$.

oxidisable sGC. We hypothesized that patients with CAD have an increased amount of oxidised SGC $[11,12]$. In agreement with this we found that the difference (Cinaciguat) - (ODQ + Cinaciguat) was significantly smaller in patients with CAD in comparison to controls with normal coronary arteries and furthermore patients with CAD had an increased oxidation index, indeed indicating a higher level of in vivo oxidised sGC in patients with CAD. Platelets as circulating blood cells will be exposed to an increased amount of ROS present in patients with atherosclerotic artery disease, which may cause an increase in the platelet pool of oxidised sGC. Inactivation of platelet SGC caused by oxidative stress has also been proposed as the potential mechanistic basis underlying the finding that patients with angiographically confirmed CAD showed decreased platelet responsiveness to the anti-aggregatory effects of NO [13,14].

The validity of our flow cytometry based assay was proven by a second assay; Western blot measuring platelet VASP phosphorylation at serine 239 , which occurs as a downstream signaling event of sGC activation in platelets (Fig. 1a) and correlates with platelet activation [8]. As Cinaciguat would only activate oxidised/haemfree sGC [1,2], we expected to see lower Western blot signals in platelet preparations from controls as compared to patients with CAD. We determined VASP phosphorylation in platelets treated with ODQ+ Cinaciguat and found a maximally increased signal in the Western blot. This was then used to calculate the ratio of (Cinaciguat)/(ODQ + Cinaciguat) to normalise for dephosphorylation of VASP, which occurs during platelet activation (data not shown [15]). We expected the ratio to be increased in patients with CAD and indeed found a significantly higher ratio as opposed to the controls. These findings complement the data that we obtained by our flow cytometry assay providing evidence for a higher oxidative burden in patients with CAD compared to individuals without CAD.

In conclusion, we are presenting novel sGC activity assays that use platelets as "oxidative memory" demonstrating increased oxidative stress in patients with CAD compared to individuals with no CAD. This assay promises to predict the response of individual patients to a therapy with direct SGC activators, allowing patient tailored pharmacotherapy. 


\section{Sources of funding}

This work was supported by the Deutsche Forschungsgemeinschaft [DFG AH 185/1-1 to I.A.], by the National Health and Medical Research Council of Australia [K.P, J.H., N.B., B.K.S., H.H.H.W.S.] and by the Cardiac Society of Australia and New Zealand [J.H.].

\section{Disclosures}

Johannes-Peter Stasch is a full-time employee of Bayer Schering Pharma AG.

\section{Appendix A. Supplementary data}

Supplementary data associated with this article can be found, in the online version, at doi:10.1016/j.atherosclerosis.2011.06.042.

\section{References}

[1] Stasch JP, Schmidt PM, Nedvetsky PI, et al. Targeting the heme-oxidized nitric oxide receptor for selective vasodilatation of diseased blood vessels. J Clin Invest 2006;116:2552-61.

[2] Evgenov OV, Pacher P, Schmidt PM, Hasko G, Schmidt HH, Stasch JP. NOindependent stimulators and activators of soluble guanylate cyclase: discovery and therapeutic potential. Nat Rev Drug Discov 2006;5:755-68.

[3] Stasch JP, Pacher P, Evgenov OV. Soluble guanylate cyclase as an emerging therapeutic target in cardiopulmonary disease. Circulation 2011;123: 2263-73.
[4] Roy B, Mo E, Vernon J, Garthwaite J. Probing the presence of the ligand-binding haem in cellular nitric oxide receptors. Br J Pharmacol 2008;153:1495-504.

[5] Boerrigter G, Costello-Boerrigter LC, Cataliotti A, Lapp H, Stasch JP, Burnett Jr JC. Targeting heme-oxidized soluble guanylate cyclase in experimental heart failure. Hypertension 2007;49:1128-33.

[6] Lapp H, Mitrovic V, Franz N, et al. Cinaciguat (BAY 58-2667) improves cardiopulmonary hemodynamics in patients with acute decompensated heart failure. Circulation 2009;119:2781-8.

[7] Walter U, Gambaryan S. cGMP and cGMP-dependent protein kinase in platelets and blood cells. Handb Exp Pharmacol 2009:533-48.

[8] Gambaryan S, Kobsar A, Hartmann S, et al. NO-synthase-/NO-independent regulation of human and murine platelet soluble guanylyl cyclase activity. J Thromb Haemost 2008;6:1376-84.

[9] Brune B, Schmidt KU, Ullrich V. Activation of soluble guanylate cyclase by carbon monoxide and inhibition by superoxide anion. Eur J Biochem 1990; $192: 683-8$

[10] Miller Jr FJ, Gutterman DD, Rios CD, Heistad DD, Davidson BL. Superoxide production in vascular smooth muscle contributes to oxidative stress and impaired relaxation in atherosclerosis. Circ Res 1998;82:1298-305

[11] Heistad DD. Oxidative stress and vascular disease: 2005 Duff lecture. Arterioscler Thromb Vasc Biol 2006;26:689-95.

[12] Stocker R, Keaney Jr JF. Role of oxidative modifications in atherosclerosis. Physiol Rev 2004;84:1381-478.

[13] Chirkov YY, Holmes AS, Chirkova LP, Horowitz JD. Nitrate resistance in platelet from patients with stable angina pectoris. Circulation 1999;100:129-34.

[14] Chirkov YY, Holmes AS, Willoughby SR, et al. Stable angina and acute coronary syndromes are associated with nitric oxide resistance in platelets. J Am Coll Cardiol 2001;37:1851-7.

[15] Jensen BO, Selheim F, Doskeland SO, Gear AR, Holmsen H. Protein kinase A mediates inhibition of the thrombin-induced platelet shape change by nitric oxide. Blood 2004;104:2775-82.

[16] Martin F, Baskaran P, Ma X, et al Structure of cinaciguat (BAY 58-2667) bound to Nostoc H-NOX domain reveals insights into heme-mimetic activation of the soluble guanylyl cyclase. J Biol Chem 2010;285:22651-7. 